\title{
SOME RESULTS ON THE CONTINUOUS DEPENDENCE OF COUPLED FIXED POINTS IN A COMPLETE METRIC SPACE
}

\author{
M. O. OLATINWO ${ }^{1, *}$ AND K. R. TIJANI ${ }^{2}$
}

\begin{abstract}
In paper of the author [M. O. Olatinwo; Stability of coupled fixed point iterations and the continuous dependence of coupled fixed points, Communications on Applied Nonlinear Analysis 19(2)(2012), 71-83], the concepts of the continuous dependence of coupled fixed points as well as the stability of coupled fixed point iterations were initiated and discussed. The contractive conditions employed were those used in [F. Sabetghadam, H. P. Masiha and A. H. Sanatpour; Some coupled fixed point theorems in cone metric spaces, Fixed Point Theory and Applications, Volume 2009, Article ID 125426, 8 Pages (2009)]. The purpose of the present article is therefore, to establish some new results on the continuous dependence of coupled fixed points by using more general contractive conditions of rational type, some of which involve strict comparison functions. An example illustrating some of the results is given to fascinate the audience of the subject being discussed in the present article.
\end{abstract}

\section{INTRODUCTION}

We consider the following definitions which shall be employed in the sequel:

Definition 1.1 [2,5,20]: Let $(X, d)$ be a metric space. An element $(x, y) \in X \times X$ is said to be a coupled fixed point of the mapping $T: X \times X \rightarrow X$ if $T(x, y)=x$ and $T(y, x)=y$.

Definition 1.2: (a) A function $\psi: \mathbb{R}_{+} \rightarrow \mathbb{R}_{+}$is called a comparison function if it satisfies the following conditions:

(i) $\psi$ is monotone increasing;

(ii) $\lim _{n \rightarrow \infty} \psi^{n}(t)=0, \forall t \geq 0$, where $\psi^{n}(t)$ denotes the $n$-th iterate of $\psi$.

(b) A comparison function satisfying $t-\psi(t) \rightarrow \infty$ as $t \rightarrow \infty$ is called a strict comparison function.

See Berinde [4], Rus [17] and Rus et al. [18] for the definition and examples of comparison functions.

Remark 1.3: Every comparison function satisfies $\psi(0)=0$.

\footnotetext{
${ }^{1}$ Department of Mathematics, Obafemi Awolowo University, Ile-Ife, Nigeria

${ }^{2}$ Department of Mathematical Sciences, Osun state University, Osogbo, Nigeria

*Corresponding aUthor

E-mail addresses: memudu.olatinwo@gmail.com/molaposi@yahoo.com/polatinwo@oauife.edu.ng, kamil_tijani2000@yahoo.com/kkrotimi72@gmail.com/kamiludeen.tijani@uniosun.edu.ng.

Key words and phrases. coupled fixed points; continuous dependence; general contractive conditions of rational type; strict comparison function.
}

Received 08/11/2021. 
Example 1.4: Consider the function $\psi: \mathbb{R}_{+} \rightarrow \mathbb{R}_{+}$defined by

$$
\psi(t)=k t, \forall t \in \mathbb{R}_{+}, k \in[0,1) .
$$

Then, $\psi$ is a strict comparison function, since

(i) $\psi(t)$ is monotone increasing;

(ii) $\lim _{n \rightarrow \infty} \psi^{n}(t)=\lim _{n \rightarrow \infty} k^{n} t=0, \forall t \geq 0$ and,

(iii) $t-\psi(t) \rightarrow \infty$ as $t \rightarrow \infty$.

Example 1.5: Define $\psi: \mathbb{R}_{+} \rightarrow \mathbb{R}_{+}$by

$$
\psi(t)=\frac{t}{1+t}, \forall t \in \mathbb{R}_{+} .
$$

Then, $\psi$ is a strict comparison function too since again,

(i) $\psi$ is monotone increasing;

(ii) $\lim _{n \rightarrow \infty} \psi^{n}(t)=\lim _{n \rightarrow \infty} \frac{t}{1+n t}=0, \forall t \geq 0$ and,

(iii) $t-\psi(t) \rightarrow \infty$ as $t \rightarrow \infty$.

Remark 1.3 (that is, $\psi(0)=0$ ) is also valid for each of both Example 1.4 and Example 1.5.

Stefan Banach [1], a great Polish Mathematician gave an abstract formulation providing a concise method for obtaining the fixed points of a map. His celebrated work gave birth to one of the fundamental theorems in functional analysis. The theorem ensures the existence and uniqueness of a fixed point under appropriate conditions. The result is stated as follows:

Theorem 1.6 (Banach's Contraction Mapping Principle [1, 4]): Let $(X, d)$ be a complete metric space and $T: X \rightarrow X$ an operator satisfying

$$
d(T x, T y) \leq a d(x, y), x, y \in X,
$$

with $a \in[0,1)$ fixed. Then;

(i) $T$ has a unique fixed point, that is $F_{T}=\left\{x^{*}\right\}$;

(ii) the Picard iteration associated to $T$, that is, the sequence $\left\{x_{n}\right\}_{n=0}^{\infty}$, defined by

$$
x_{n}=T\left(x_{n-1}\right)=T^{n}\left(x_{0}\right), n=1,2, \cdots,
$$

converges to $x^{*}$, for any initial guess $x_{0} \in X$;

(iii) the a priori and a posteriori error estimates

$$
d\left(x_{n}, x^{*}\right) \leq \frac{a^{n}}{1-a} \cdot d\left(x_{0}, x_{1}\right), n=1,2, \cdots
$$

and

hold respectively, and

$$
d\left(x_{n}, x^{*}\right) \leq \frac{a}{1-a} \cdot d\left(x_{n-1}, x_{n}\right), n=1,2, \cdots
$$

(iv) the rate of convergence is given by

$$
d\left(x_{n}, x^{*}\right) \leq \operatorname{ad}\left(x_{n-1}, x^{*}\right) \leq a^{2} d\left(x_{n-2}, x^{*}\right) \leq \cdots \leq a^{n} d\left(x_{0}, x^{*}\right),(n=1,2, \cdots) .
$$

The condition (1) is called the Banach's contractive condition and the Picard iteration (2) was employed to approximate the fixed point $x^{*}$ of $T$. 
In many applications, the operator $T$ in the Picard iteration (2) may depend on additional parameter $\lambda \in Y$, where $Y$ is a parameter space (or, in a more general context, $Y$ is a topological space). Therefore, the fixed point iterative procedure (2) is replaced by

$$
x_{\lambda}=S_{\lambda} x_{\lambda}, x_{\lambda} \in X
$$

Let $(X, d)$ be a complete metric space, $(Y, \tau)$ a topological space and $S: X \times Y \rightarrow X$ a mapping depending on the parameter $\lambda \in Y$. Suppose that $S_{\lambda}=S(x, \lambda), x \in X, \lambda \in Y$, has a unique fixed point $x_{\lambda}^{*} \in X$, for any $\lambda \in Y$. We define the mapping $U: Y \rightarrow X$ by $U(\lambda)=x_{\lambda}^{*}, \forall \lambda \in Y$. Our interest lies in obtaining sufficient conditions on $S_{\lambda}$ that guarantee the continuity of the mapping $U$.

Some authors in fixed point theory have worked on the continuous dependence of fixed points. Condition (1) was employed in Zeidler [22] to prove a result on the continuous dependence of the fixed point on a parameter. Also, in Olatinwo [8], the notion of stability of the fixed point (that is, continuous dependence of the fixed points on a parameter) has been extended from the complete metric space to the normed linear space setting for Schaefer and Mann iterative algorithms, and this article contains the pioneering work on the continuous dependence of the fixed points in Banach space and normed linear space. For more detail on the contributions of authors on the concept of continuous dependence of fixed point, we refer to Berinde [4], Rus and Rus [15], Rus and Muresan [16] as well as some others in the literature. The study of the continuous dependence of the fixed points has been extended to the coupled fixed point setting in Olatinwo [12].

The continuous dependence of the coupled fixed point has been formulated in the following general context in [12]:

Let $(X, d)$ be a complete metric space, $(Y, \tau)$ a topological space and $T: X \times X \times Y \rightarrow X$ a mapping depending on the parameter $\lambda \in Y$. The operator $T$ in the coupled iteration may depend on additional parameter $\lambda \in Y$, where $Y$ is a parameter space (or, in a more general context, $Y$ is a topological space).

Therefore, the coupled fixed point iterative procedure $\left\{\left(x_{n}, y_{n}\right)\right\}_{n=0}^{\infty} \subset X \times X$ defined by

$$
x_{n+1}=T\left(x_{n}, y_{n}\right), y_{n+1}=T\left(y_{n}, x_{n}\right), n=0,1,2, \cdots,
$$

is replaced by

$$
x_{\lambda}=T_{\lambda}\left(x_{\lambda}, y_{\lambda}\right), y_{\lambda}=T_{\lambda}\left(y_{\lambda}, x_{\lambda}\right), x_{\lambda}, y_{\lambda} \in Y .
$$

Suppose that $T_{\lambda}=T(x, y, \lambda), x, y \in X, \lambda \in Y$, has a unique coupled fixed point $\left(x_{\lambda}^{*}, y_{\lambda}^{*}\right) \in$ $X \times X$, for any $\lambda \in Y$.

We define the mappings $U, V: Y \rightarrow X$ by $U(\lambda)=x_{\lambda}^{*}, V(\lambda)=y_{\lambda}^{*}, \forall \lambda \in Y$.

We are interested in obtaining sufficient conditions on $S_{\lambda}$ that guarantee the continuity of the mappings $U, V: Y \rightarrow X$.

We also refer to the articles of the author [9,10,13] for various results on the notion of the continuous dependence of fixed points for different iterative processes and different contractive conditions.

Furthermore, in this paper, we present some results on the continuous dependence of coupled fixed point in complete metric spaces using rational type contractive conditions. 


\section{MAin Results}

Theorem 2.1: Let $(X, d)$ be a complete metric space and $(Y, \tau)$ a topological space. Let $T: X \times X \times Y \rightarrow X$ be a continuous mapping satisfying the contractive condition of rational type

$$
\begin{aligned}
d(T(x, y, \lambda), T(u, v, \lambda)) \leq & \alpha \frac{[d(x, T(u, v, \lambda))]^{q} \cdot d(x, T(x, y, \lambda)) \cdot d(u, T(u, v, \lambda))}{d(x, u)+\gamma d(u, T(u, v, \lambda))} \\
& +\psi(d(x, u)), \forall x, y, u, v \in X,(4)
\end{aligned}
$$

where $d(x, u)+\gamma d\left(u, T(u, v, \lambda)>0, q \geq 0, \alpha \geq 0, \gamma \geq 0\right.$ and $\psi: \mathbb{R}_{+} \rightarrow \mathbb{R}_{+}$is a strict comparison function. Let $\left(x_{\lambda}^{*}, y_{\lambda}^{*}\right)$ be the unique coupled fixed point of $T_{\lambda}$ defined in $(C 2)$. Suppose that $\left\{\left(x_{n}, y_{n}\right)\right\}_{n=0}^{\infty} \subset X \times X$ is the coupled fixed point iterative procedure defined by $(C 1)$. Then, $U, V: Y \rightarrow X$ defined by $U(\lambda)=x_{\lambda}^{*}, V(\lambda)=y_{\lambda}^{*}, x_{\lambda}^{*}, y_{\lambda}^{*} \in X, \lambda \in Y$ are continuous mappings.

Proof: Let $\lambda_{1}, \lambda_{2} \in Y$. Since $\left(x_{\lambda}^{*}, y_{\lambda}^{*}\right)$ is a unique coupled fixed point of $T_{\lambda}$, we have that $x_{\lambda}^{*}=T\left(x_{\lambda}^{*}, y_{\lambda}^{*}, \lambda\right), y_{\lambda}^{*}=T\left(y_{\lambda}^{*}, x_{\lambda}^{*}, \lambda\right)$.

By Condition (4), we have that

$$
\begin{aligned}
d\left(x_{\lambda_{1}}^{*}, x_{\lambda_{2}}^{*}\right) & =d\left(T\left(x_{\lambda_{1}}^{*}, y_{\lambda_{1}}^{*}, \lambda_{1}\right), T\left(x_{\lambda_{2}}^{*}, y_{\lambda_{2}}^{*}, \lambda_{2}\right)\right), \\
\leq & d\left(T\left(x_{\lambda_{1}}^{*}, y_{\lambda_{1}}^{*}, \lambda_{1}\right), T\left(x_{\lambda_{2}}^{*}, y_{\lambda_{2}}^{*}, \lambda_{1}\right)\right)+d\left(T\left(x_{\lambda_{2}}^{*}, y_{\lambda_{2}}^{*}, \lambda_{1}\right)+T\left(x_{\lambda_{2}}^{*}, y_{\lambda_{2}}^{*}, \lambda_{2}\right)\right) \\
\leq & \alpha \frac{\left[d\left(x_{\lambda_{1}}^{*}, T\left(x_{\lambda_{2}}^{*}, y_{\lambda_{2}}^{*},\right)\right]^{q}, d\left(x_{\lambda_{1}}^{*}, T\left(x_{\lambda_{1}}^{*}, y_{\lambda_{1}}^{*}, \lambda_{1}\right)\right) \cdot d\left(x_{\lambda_{2}}^{*}, T\left(x_{\lambda_{2}}^{*}, y_{\lambda_{2}}^{*}, \lambda_{1}\right)\right)\right.}{d\left(x_{\lambda_{1}}^{*}, x_{\lambda_{2}}^{*}\right)+\gamma d\left(x_{\lambda_{2}}^{*}, T\left(x_{\lambda_{2}}^{*}, y_{\lambda_{2}}^{*}, \lambda_{1}\right)\right)} \\
& +\psi\left(d\left(x_{\lambda_{1}}^{*}, x_{\lambda_{2}}^{*}\right)\right)+d\left(T_{\lambda_{1}}\left(x_{\lambda_{2}}^{*}, y_{\lambda_{2}}^{*}\right), T_{\lambda_{2}}\left(x_{\lambda_{2}}^{*}, y_{\lambda_{2}}^{*}\right)\right) \\
& =\alpha \frac{\left[d\left(x_{\lambda_{2}}^{*}, T\left(x_{\lambda_{2}}^{*}, y_{\lambda_{2}}^{*}, \lambda_{1}\right)\right)\right]^{q} \cdot d\left(x_{\lambda_{1}}^{*}, x_{\lambda_{1}}^{*}\right), d\left(x_{\lambda_{2}}^{*}, T\left(x_{\lambda_{2}}^{*}, y_{\lambda_{2}}^{*}, \lambda_{1}\right)\right)}{d\left(x_{\lambda_{1}}^{*}, x_{\lambda_{2}}^{*}\right)+\gamma d\left(x_{\lambda_{2}}^{*}, T\left(x_{\lambda_{2}}^{*}, y_{\lambda_{2}}^{*}, \lambda_{1}\right)\right)} \\
& +\psi\left(d\left(x_{\lambda_{1}}^{*}, x_{\lambda_{2}}^{*}\right)\right)+d\left(T_{\lambda_{1}}\left(x_{\lambda_{2}}^{*}, y_{\lambda_{2}}^{*}\right), T_{\lambda_{2}}\left(x_{\lambda_{2}}^{*}, y_{\lambda_{2}}^{*}\right)\right) \\
& =\psi\left(d\left(x_{\lambda_{1}}^{*}, x_{\lambda_{2}}^{*}\right)\right)+d\left(T_{\lambda_{1}}\left(x_{\lambda_{2}}^{*}, y_{\lambda_{2}}^{*}\right), T_{\lambda_{2}}\left(x_{\lambda_{2}}^{*}, y_{\lambda_{2}}^{*}\right)\right)(5)
\end{aligned}
$$

By Condition (4) again, we have

$$
\begin{aligned}
d\left(y_{\lambda_{1}}^{*}, y_{\lambda_{2}}^{*}\right) & =d\left(T\left(y_{\lambda_{1}}^{*}, x_{\lambda_{1}}^{*}, \lambda_{1}\right), T\left(y_{\lambda_{2}}^{*}, x_{\lambda_{2}}^{*}, \lambda_{2}\right)\right), \\
\leq & d\left(T\left(y_{\lambda_{1}}^{*}, x_{\lambda_{1}}^{*}, \lambda_{1}\right), T\left(y_{\lambda_{2}}^{*}, x_{\lambda_{2}}^{*}, \lambda_{1}\right)\right)+d\left(T\left(y_{\lambda_{2}}^{*}, x_{\lambda_{2}}^{*}, \lambda_{1}\right)+T\left(y_{\lambda_{2}}^{*}, x_{\lambda_{2}}^{*}, \lambda_{2}\right)\right) \\
\leq & \alpha \frac{\left[d\left(y_{\lambda_{1}}^{*}, T\left(y_{\lambda_{2}}^{*}, x_{\lambda_{2}}^{*}, \lambda_{1}\right)\right)\right]^{q} \cdot d\left(y_{\lambda_{1}}^{*}, T\left(y_{\lambda_{1}}, x_{\lambda_{1}}^{*}, \lambda_{1}\right)\right) \cdot d\left(y_{\lambda_{2}}^{*}, T\left(y_{\lambda_{2}}^{*}, x_{\lambda_{2}}^{*}, \lambda_{1}\right)\right)}{d\left(y_{\lambda_{1}}^{*}, y_{\lambda_{2}}^{*}\right)+\gamma d\left(y_{\lambda_{2}}^{*}, T\left(y_{\lambda_{2}}^{*}, x_{\lambda_{2}}^{*}, \lambda_{1}\right)\right)} \\
& +\psi\left(d\left(y_{\lambda_{1}}^{*}, y_{\lambda_{2}}^{*}\right)\right)+d\left(T_{\lambda_{1}}^{*}\left(y_{\lambda_{2}}^{*}, x_{\lambda_{2}}^{*}\right), T_{\lambda_{2}}\left(y_{\lambda_{2}}^{*}, x_{\lambda_{2}}^{*}\right)\right) \\
& =\alpha \frac{\left[d\left(y_{\lambda_{1}}^{*}, T\left(y_{\lambda_{2}}^{*}, x_{\lambda_{2}}^{*}, \lambda_{1}\right)\right)\right]^{q} \cdot d\left(y_{\lambda_{1}}^{*}, y_{\lambda_{1}}^{*}\right) \cdot d\left(y_{\lambda_{2}}^{*}, T\left(y_{\lambda_{2}}^{*}, x_{\lambda_{2}}^{*}, \lambda_{1}\right)\right)}{d\left(y_{\lambda_{1}}^{*}, y_{\lambda_{2}}^{*}\right)+\gamma d\left(y_{\lambda_{2}}^{*}, T\left(y_{\lambda_{2}}^{*}, x_{\lambda_{2}}^{*}, \lambda_{1}\right)\right)} \\
& +\psi\left(d\left(y_{\lambda_{1}}^{*}, y_{\lambda_{2}}^{*}\right)\right)+d\left(T_{\lambda_{1}}\left(y_{\lambda_{2}}^{*}, x_{\lambda_{2}}^{*}\right), T_{\lambda_{2}}\left(y_{\lambda_{2}}^{*}, x_{\lambda_{2}}^{*}\right)\right) \\
& =\psi\left(d\left(y_{\lambda_{1}}^{*}, y_{\lambda_{2}}^{*}\right)\right)+d\left(T_{\lambda_{1}}\left(y_{\lambda_{2}}^{*}, x_{\lambda_{2}}^{*}\right), T_{\lambda_{2}}\left(y_{\lambda_{2}}^{*}, x_{\lambda_{2}}^{*}\right)\right) .(6)
\end{aligned}
$$

Adding (5) and (6) yields

$$
\begin{aligned}
{\left[d\left(x_{\lambda_{1}}^{*}, x_{\lambda_{2}}^{*}\right)-\psi\left(d\left(x_{\lambda_{1}}^{*}, x_{\lambda_{2}}^{*}\right)\right)\right] } & +\left[d\left(y_{\lambda_{1}}^{*}, y_{\lambda_{2}}^{*}\right)-\psi\left(d\left(y_{\lambda_{1}}^{*}, y_{\lambda_{2}}^{*}\right)\right)\right] \leq d\left(T_{\lambda_{1}}\left(x_{\lambda_{2}}^{*}, y_{\lambda_{2}}^{*}\right), T_{\lambda_{2}}\left(x_{\lambda_{2}}^{*}, y_{\lambda_{2}}^{*}\right)\right) \\
& +d\left(T_{\lambda_{1}}\left(y_{\lambda_{2}}^{*}, x_{\lambda_{2}}^{*}\right), T_{\lambda_{2}}\left(y_{\lambda_{2}}^{*}, x_{\lambda_{2}}^{*}\right)\right) .
\end{aligned}
$$

Since $T$ is continuous and $\psi$ is a strict comparison function, we have $d\left(T_{\lambda_{1}}\left(x_{\lambda_{2}}^{*}, y_{\lambda_{2}}^{*}\right), T_{\lambda_{2}}\left(x_{\lambda_{2}}^{*}, y_{\lambda_{2}}^{*}\right)\right)+d\left(T_{\lambda_{1}}\left(y_{\lambda_{2}}^{*}, x_{\lambda_{2}}^{*}\right), T_{\lambda_{2}}\left(y_{\lambda_{2}}^{*}, x_{\lambda_{2}}^{*}\right)\right) \rightarrow 0$ as $\lambda_{2} \rightarrow \lambda_{1}$.

Therefore, we obtain from $(\star \star)$ that $\left[d\left(x_{\lambda_{1}}^{*}, x_{\lambda_{2}}^{*}\right)-\psi\left(d\left(x_{\lambda_{1}}^{*}, x_{\lambda_{2}}^{*}\right)\right)\right]+\left[d\left(y_{\lambda_{1}}^{*}, y_{\lambda_{2}}^{*}\right)-\psi\left(d\left(y_{\lambda_{1}}^{*}, y_{\lambda_{2}}^{*}\right)\right)\right] \rightarrow 0$ as $\lambda_{2} \rightarrow \lambda_{1}$, from which it follows that $d\left(x_{\lambda_{1}}^{*}, x_{\lambda_{2}}^{*}\right)-\psi\left(d\left(x_{\lambda_{1}}^{*}, x_{\lambda_{2}}^{*}\right)\right) \rightarrow 0$ as $\lambda_{2} \rightarrow \lambda_{1}$, yielding $d\left(x_{\lambda_{1}}^{*}, x_{\lambda_{2}}^{*}\right) \rightarrow 0$ as $\lambda_{2} \rightarrow \lambda_{1}$. Also, $d\left(y_{\lambda_{1}}^{*}, y_{\lambda_{2}}^{*}\right)-\psi\left(d\left(y_{\lambda_{1}}^{*}, y_{\lambda_{2}}^{*}\right)\right) \rightarrow 0$ as $\lambda_{2} \rightarrow \lambda_{1}$ gives $d\left(y_{\lambda_{1}}^{*}, y_{\lambda_{2}}^{*}\right) \rightarrow 0$ as $\lambda_{2} \rightarrow \lambda_{1}$. 
Hence, $d\left(x_{\lambda_{1}}^{*}, x_{\lambda_{2}}^{*}\right) \rightarrow 0$ as $\lambda_{2} \rightarrow \lambda_{1}$ implies $d\left(U\left(\lambda_{1}\right), U\left(\lambda_{2}\right)\right) \rightarrow 0$ as $\lambda_{2} \rightarrow \lambda_{1}$, and also

$d\left(y_{\lambda_{1}}^{*}, y_{\lambda_{2}}^{*}\right) \rightarrow 0$ as $\lambda_{2} \rightarrow \lambda_{1}$ implies $d\left(V\left(\lambda_{1}\right), V\left(\lambda_{2}\right)\right) \rightarrow 0$ as $\lambda_{2} \rightarrow \lambda_{1}$.

Therefore, the mappings $U, V: Y \rightarrow X$ are continuous.

The following result is a corollary of Theorem 2.1:

Corollary 2.2: Let $(X, d)$ be a complete metric space and $(Y, \tau)$ a topological space. Let $T: X \times X \times Y \rightarrow X$ be a continuous mapping satisfying the contractive condition of rational type

$$
\begin{aligned}
d(T(x, y, \lambda), T(u, v, \lambda)) \leq & \alpha \frac{[d(x, T(u, v, \lambda))]^{q} \cdot d(x, T(x, y, \lambda)) \cdot d(u, T(u, v, \lambda))}{d(x, u)+\gamma d(u, T(u, v, \lambda))} \\
& +\beta d(x, u)), \quad \forall x, y, u, v \in X,(7)
\end{aligned}
$$

$d(x, u)+\gamma d(u, T(u, v, \lambda)>0$, where $q, \alpha, \gamma \geq 0$ and $\beta \in[0,1)$.

Let $\left(x_{\lambda}^{*}, y_{\lambda}^{*}\right)$ be the unique coupled fixed point of $T_{\lambda}$ defined in $(C 2)$. Suppose that $\left\{\left(x_{n}, y_{n}\right)\right\}_{n=0}^{\infty} \subset X \times X$ is the coupled fixed point iterative procedure defined by $(C 1)$. Then, $U, V: Y \rightarrow X$ defined by $U(\lambda)=x_{\lambda}^{*}, V(\lambda)=y_{\lambda}^{*}, x_{\lambda}^{*}, y_{\lambda}^{*} \in X, \lambda \in Y$ are continuous mappings.

Proof: Let $\lambda_{1}, \lambda_{2} \in Y$. Since $\left(x_{\lambda}^{*}, y_{\lambda}^{*}\right)$ is a unique coupled fixed point of $T_{\lambda}$, we have that $x_{\lambda}^{*}=T\left(x_{\lambda}^{*}, y_{\lambda}^{*}, \lambda\right), y_{\lambda}^{*}=T\left(y_{\lambda}^{*}, x_{\lambda}^{*}, \lambda\right)$.

By using Condition (7), we have that

$$
d\left(x_{\lambda_{1}}^{*}, x_{\lambda_{2}}^{*}\right) \leq \frac{1}{1-\beta} d\left(T_{\lambda_{1}}\left(x_{\lambda_{2}}^{*}, y_{\lambda_{2}}^{*}\right), T_{\lambda_{2}}\left(x_{\lambda_{2}}^{*}, y_{\lambda_{2}}^{*}\right)\right),
$$

and

$$
d\left(y_{\lambda_{1}}^{*}, y_{\lambda_{2}}^{*}\right) \leq \frac{1}{1-\beta} d\left(T_{\lambda_{1}}\left(y_{\lambda_{2}}^{*}, x_{\lambda_{2}}^{*}\right), T_{\lambda_{2}}\left(y_{\lambda_{2}}^{*}, x_{\lambda_{2}}^{*}\right)\right) .
$$

Adding (8) and (9) gives

$$
\begin{aligned}
d\left(x_{\lambda_{1}}^{*}, x_{\lambda_{2}}^{*}\right)+d\left(y_{\lambda_{1}}^{*}, y_{\lambda_{2}}^{*}\right) \leq & \frac{1}{1-\beta}\left[d\left(T_{\lambda_{1}}\left(x_{\lambda_{2}}^{*}, y_{\lambda_{2}}^{*}\right), T_{\lambda_{2}}\left(x_{\lambda_{2}}^{*}, y_{\lambda_{2}}^{*}\right)\right)\right. \\
& \left.+d\left(T_{\lambda_{1}}\left(y_{\lambda_{2}}^{*}, x_{\lambda_{2}}^{*}\right), T_{\lambda_{2}}\left(y_{\lambda_{2}}^{*}, x_{\lambda_{2}}^{*}\right)\right)\right] \rightarrow 0 \text { as } \lambda_{2} \rightarrow \lambda_{1},
\end{aligned}
$$

since $T$ is continuous.

Hence,

$$
d\left(x_{\lambda_{1}}^{*}, x_{\lambda_{2}}^{*}\right)+d\left(y_{\lambda_{1}}^{*}, y_{\lambda_{2}}^{*}\right) \rightarrow 0 \text { as } \lambda_{2} \rightarrow \lambda_{1} .
$$

That is, $d\left(x_{\lambda_{1}}^{*}, x_{\lambda_{2}}^{*}\right) \rightarrow 0$ as $\lambda_{2} \rightarrow \lambda_{1}$,

so that $d\left(U\left(\lambda_{1}\right), U\left(\lambda_{2}\right)\right) \rightarrow 0$ as $\lambda_{2} \rightarrow \lambda_{1}$.

Similarly, $d\left(y_{\lambda_{1}}^{*}, y_{\lambda_{2}}^{*}\right) \rightarrow 0$ as $\lambda_{2} \rightarrow \lambda_{1}$,

so that $d\left(V\left(\lambda_{1}\right), V\left(\lambda_{2}\right)\right) \rightarrow 0$ as $\lambda_{2} \rightarrow \lambda_{1}$.

Therefore, the mappings $U, V: Y \rightarrow X$ as defined are continuous.

Theorem 2.3: Let $(X, d)$ be a complete metric space and $(Y, \tau)$ a topological space. Let $T: X \times X \times Y \rightarrow X$ be a continuous mapping satisfying the contractive condition of rational type

$$
\begin{aligned}
d(T(x, y, \lambda), T(u, v, \lambda)) \leq & \alpha\left(\frac{d(x, T(u, v, \lambda)) \cdot d(x, T(x, y, \lambda)) \cdot d(u, T(u, v, \lambda))}{d(x, u)+d(u, T(u, v, \lambda))}\right) \\
& +\psi(d(x, u)), \quad \forall x, y, u, v \in X(10)
\end{aligned}
$$


where $d(x, u)+d\left(u, T(u, v, \lambda)>0, \alpha: \mathbb{R}_{+} \rightarrow \mathbb{R}_{+}\right.$is a monotone increasing function such that $\alpha(0)=0$ and $\psi: \mathbb{R}_{+} \rightarrow \mathbb{R}_{+}$is a strict comparison function. Let $\left(x_{\lambda}^{*}, y_{\lambda}^{*}\right)$ be the unique coupled fixed point of $T_{\lambda}$ defined in (C2). Suppose that $\left\{\left(x_{n}, y_{n}\right)\right\}_{n=0}^{\infty} \subset X \times X$ is the coupled fixed point iterative procedure defined by $(C 1)$. Then, $U, V: Y \rightarrow X$ defined by $U(\lambda)=x_{\lambda}^{*}, V(\lambda)=y_{\lambda}^{*}, x_{\lambda}^{*}, y_{\lambda}^{*} \in X, \lambda \in Y$ are continuous mappings.

Proof: Let $\lambda_{1}, \lambda_{2} \in Y$. Since $\left(x_{\lambda}^{*}, y_{\lambda}^{*}\right)$ is a unique coupled fixed point of $T_{\lambda}$, we have that $x_{\lambda}^{*}=T\left(x_{\lambda}^{*}, y_{\lambda}^{*}, \lambda\right), y_{\lambda}^{*}=T\left(y_{\lambda}^{*}, x_{\lambda}^{*}, \lambda\right)$.

By Condition (10), we have that

$$
\begin{aligned}
d\left(x_{\lambda_{1}}^{*}, x_{\lambda_{2}}^{*}\right) & =d\left(T\left(x_{\lambda_{1}}^{*}, y_{\lambda_{1}}^{*}, \lambda_{1}\right), T\left(x_{\lambda_{2}}^{*}, y_{\lambda_{2}}^{*}, \lambda_{2}\right)\right), \\
& \leq d\left(T\left(x_{\lambda_{1}}^{*}, y_{\lambda_{1}}^{*}, \lambda_{1}\right), T\left(x_{\lambda_{2}}^{*}, y_{\lambda_{2}}^{*}, \lambda_{1}\right)\right)+d\left(T\left(x_{\lambda_{2}}^{*}, y_{\lambda_{2}}^{*}, \lambda_{1}\right)+T\left(x_{\lambda_{2}}^{*}, y_{\lambda_{2}}^{*}, \lambda_{2}\right)\right) \\
& \leq \alpha\left(\frac{d\left(x_{\lambda_{1}}^{*}, T\left(x_{\lambda_{2}}^{*}, y_{\lambda_{2}}^{*}, \lambda_{1}\right)\right) \cdot d\left(x_{\lambda_{1}}^{*}, T\left(x_{\lambda_{1}}^{*}, y_{\lambda_{1}}^{*}, \lambda_{1}\right)\right) \cdot d\left(x_{\lambda_{2}}^{*}, T\left(x_{\lambda_{2}}^{*}, y_{\lambda_{2}}^{*}, \lambda_{1}\right)\right)}{d\left(x_{\lambda_{1}}^{*}, x_{\lambda_{2}}^{*}\right)+d\left(x_{\lambda_{2}}^{*}, T\left(x_{\lambda_{2}}^{*}, y_{\lambda_{2}}^{*}, \lambda_{1}\right)\right)}\right) \\
+ & \psi\left(d\left(x_{\lambda_{1}}^{*}, x_{\lambda_{\lambda}}^{*}\right)\right)+d\left(T_{\lambda_{1}}\left(x_{\lambda_{2}}^{*}, y_{\lambda_{2}}^{*}\right), T_{\lambda_{2}}\left(x_{\lambda_{2}}^{*}, y_{\lambda_{2}}^{*}\right)\right) \\
= & \alpha\left(\frac{d\left(x_{\lambda_{2}}^{*}, T\left(x_{\lambda_{2}}^{*}, y_{\lambda_{2}}^{*}, \lambda_{1}\right)\right) \cdot d\left(x_{\lambda_{1}}^{*}, x_{\lambda_{1}}^{*}\right) \cdot d\left(x_{\lambda_{2}}^{*}, T\left(x_{\lambda_{2}}^{*}, y_{\lambda_{2}}^{*}, \lambda_{1}\right)\right)}{d\left(x_{\lambda_{1}}^{*}, x_{\lambda_{2}}^{*}\right)+d\left(x_{\lambda_{2}}^{*}, T\left(x_{\lambda_{2}}^{*}, y_{\lambda_{2}}^{*}, \lambda_{1}\right)\right)}\right)+ \\
& \psi\left(d\left(x_{\lambda_{1}}^{*}, x_{\lambda_{2}}^{*}\right)\right)+d\left(T_{\lambda_{1}}\left(x_{\lambda_{2}}^{*}, y_{\lambda_{2}}^{*}\right), T_{\lambda_{2}}\left(x_{\lambda_{2}}^{*}, y_{\lambda_{2}}^{*}\right)\right) \\
= & \psi\left(d\left(x_{\lambda_{1}}^{*}, x_{\lambda_{2}}^{*}\right)\right)+d\left(T_{\lambda_{1}}\left(x_{\lambda_{2}}^{*}, y_{\lambda_{2}}^{*}\right), T_{\lambda_{2}}\left(x_{\lambda_{2}}^{*}, y_{\lambda_{2}}^{*}\right)\right), \text { since } \alpha(0)=0 .(11)
\end{aligned}
$$

Again, by Condition (10), we have

$$
\begin{aligned}
d\left(y_{\lambda_{1}}^{*}, y_{\lambda_{2}}^{*}\right) & =d\left(T\left(y_{\lambda_{1}}^{*}, x_{\lambda_{1}}^{*}, \lambda_{1}\right), T\left(y_{\lambda_{2}}^{*}, x_{\lambda_{2}}^{*}, \lambda_{2}\right)\right), \\
& \leq d\left(T\left(y_{\lambda_{1}}^{*}, x_{\lambda_{1}}^{*}, \lambda_{1}\right), T\left(y_{\lambda_{2}}^{*}, x_{\lambda_{2}}^{*}, \lambda_{1}\right)\right)+d\left(T\left(y_{\lambda_{2}}^{*}, x_{\lambda_{2}}^{*}, \lambda_{1}\right)+T\left(y_{\lambda_{2}}^{*}, x_{\lambda_{2}}^{*}, \lambda_{2}\right)\right) \\
& \leq \alpha\left(\frac{d\left(y_{\lambda_{1}}^{*}, T\left(y_{\lambda_{2}}^{*}, x_{\lambda_{2}}^{*}, \lambda_{1}\right)\right) \cdot d\left(y_{\lambda_{1}}^{*}, T\left(y_{\lambda_{1}}^{*}, x_{\lambda_{1}}^{*}, \lambda_{1}\right)\right) \cdot d\left(y_{\lambda_{2}}^{*}, T\left(y_{\lambda_{2}}^{*}, x_{\lambda_{2}}^{*}, \lambda_{1}\right)\right)}{d\left(y_{\lambda_{1}}^{*}, y_{\lambda_{2}}^{*}\right)+d\left(y_{\lambda_{2}}^{*}, T\left(y_{\lambda_{2}}^{*}, x_{\lambda_{2}}^{*}, \lambda_{1}\right)\right)}\right) \\
& +\psi\left(d\left(y_{\lambda_{1}}^{*}, y_{\lambda_{2}}^{*}\right)\right)+d\left(T_{\lambda_{1}}\left(y_{\lambda_{2}}^{*}, x_{\lambda_{2}}^{*}\right), T_{\lambda_{2}}\left(y_{\lambda_{2}}^{*}, x_{\lambda_{2}}^{*}\right)\right) \\
& =\alpha\left(\frac{d\left(y_{\lambda_{1}}^{*}, T\left(y_{\lambda_{2}}^{*}, x_{\lambda_{2}}^{*}, \lambda_{1}\right) \cdot d\left(y_{\lambda_{1}}^{*}, y_{\lambda_{1}}^{*}\right) \cdot d\left(y_{\lambda_{2}}^{*}, T\left(y_{\lambda_{2}}^{*}, x_{\lambda_{2}}^{*}, \lambda_{1}\right)\right)\right.}{d\left(y_{\lambda_{1}}^{*}, y_{\lambda_{2}}^{*}\right)+d\left(y_{\lambda_{2}}^{*}, T\left(y_{\lambda_{2}}^{*}, x_{\lambda_{2}}^{*}, \lambda_{1}\right)\right)}\right. \\
& +\psi\left(d\left(y_{\lambda_{1}}^{*}, y_{\lambda_{2}}^{*}\right)\right)+d\left(T_{\lambda_{1}}\left(y_{\lambda_{2}}^{*}, x_{\lambda_{\lambda}}^{*}\right), T_{\lambda_{2}}\left(y_{\lambda_{2}}^{*}, x_{\lambda_{2}}^{*}\right)\right) \\
& =\psi\left(d\left(y_{\lambda_{1}}^{*}, y_{\lambda_{2}}^{*}\right)\right)+d\left(T_{\lambda_{1}}\left(y_{\lambda_{2}}^{*}, x_{\lambda_{2}}^{*}\right), T_{\lambda_{2}}\left(y_{\lambda_{2}}^{*}, x_{\lambda_{2}}^{*}\right)\right), \text { since } \alpha(0)=0 .(12)
\end{aligned}
$$

Adding (11) and (12) yields inequality ( $\star \star)$ as in the proof of Theorem 2.1.

Thus, since $T$ is continuous and $\psi$ is a strict comparison function, we have as in the proof of Theorem 2.2 that

$d\left(U\left(\lambda_{1}\right), U\left(\lambda_{2}\right)\right) \rightarrow 0$ as $\lambda_{2} \rightarrow \lambda_{1}$ and $d\left(V\left(\lambda_{1}\right), V\left(\lambda_{2}\right)\right) \rightarrow 0$ as $\lambda_{2} \rightarrow \lambda_{1}$.

That is, the mappings $U, V: Y \rightarrow X$ are continuous.

Theorem 2.4 Let $(X, d)$ be a complete metric space and $(Y, \tau)$ a topological space. Let $T: X \times X \times Y \rightarrow X$ be a continuouos mapping satisfying the contractive condition of rational type

$$
d(T(x, y, \lambda), T(u, v, \lambda)) \leq \alpha\left(\frac{d(x, T(x, y, \lambda)) \cdot d(u, T(u, v, \lambda))}{d(x, u)}\right)+\psi(d(x, u)),
$$

$\forall x, y, u, v \in X, d(x, u)>0$, where $\alpha: \mathbb{R}_{+} \rightarrow \mathbb{R}_{+}$is a monotone increasing function such that $\alpha(0)=0$ and $\psi: \mathbb{R}_{+} \rightarrow \mathbb{R}_{+}$is a continuous, strict comparison function. Let $\left(x_{\lambda}^{*}, y_{\lambda}^{*}\right)$ be the unique coupled fixed point of $T_{\lambda}$ defined in (C2). Suppose that $\left\{\left(x_{n}, y_{n}\right)\right\}_{n=0}^{\infty} \subset X \times X$ is the coupled fixed point iterative procedure defined by $(C 1)$.

Then, $U, V: Y \rightarrow X$ defined by $U(\lambda)=x_{\lambda}^{*}, V(\lambda)=y_{\lambda}^{*}, x_{\lambda}^{*}, y_{\lambda}^{*} \in X, \lambda \in Y$ are continuous 
mappings.

Proof: The proof of this result follows similar line of argument as in Theorem 2.3.

Remark 2.5: Theorem 2.1 - Theorem 2.4 (including Corollary 2.2) contained in the present article generalize Theorem 3.1 - Theorem 3.6 established in Olatinwo [12]. Furthermore, Theorem 2.1, Theorem 2.3 as well as Theorem 2.4 are generalizations and extensions of Theorem 7.7 in Berinde [4] (which is itself Theorem 7.1.2 in Rus [17]).

Consider the following example regarding Theorem 2.1:

Example 2.6: Let $T_{\lambda}(x, y)=T(x, y, \lambda)$ in the contractive condition (4) and suppose that $X=C[a, b]=\{h \in X \mid h:[a, b] \rightarrow \mathbb{R}\}=$ space of all real-valued continuous bounded functions on $[a, b] \subset Y=\mathbb{R}$ with the usual topology.

Define $T: X \times X \times Y \rightarrow X$ by $T_{\lambda}\left(x_{\mu}, y_{\eta}\right)=e^{-\lambda}-y_{\eta}$, where $x_{\mu}, y_{\eta} \in X, \lambda, \mu, \eta \in[a, b]$.

Let $\psi: \mathbb{R}^{+} \rightarrow \mathbb{R}^{+}$defined by $\psi(t)=\frac{3}{4} t, t \in \mathbb{R}^{+}$, be a given strict comparison function. Then, $\left(\frac{e^{-\lambda}}{3}, \frac{2 e^{-\lambda}}{3}\right)$ is the the unique coupled fixed point of $T_{\lambda}$ and the mappings $U, V:[a, b] \rightarrow X$ defined by $U(\lambda)=x_{\lambda}^{*}, V(\lambda)=y_{\lambda}^{*}, x_{\lambda}^{*}, y_{\lambda}^{*} \in X, \lambda \in[a, b]$ are continuous, where $\left(x_{\lambda}^{*}, y_{\lambda}^{*}\right)$ is the unique coupled fixed point of $T_{\lambda}$.

Solution: Assume the usual metric on $\mathbb{R}$ (that is, $d(u, v)=|u-v|, \quad u, v \in \mathbb{R}$ ). Indeed, $\psi: \mathbb{R}^{+} \rightarrow \mathbb{R}^{+}$defined by $\psi(t)=\frac{3}{4} t, t \in \mathbb{R}^{+}$, is a strict comparison function.

Recall that in a proof line of Theorem 2.1, we have the Inequality $(\star \star)$ as follows:

$$
\begin{aligned}
{\left[d\left(x_{\lambda_{1}}^{*}, x_{\lambda_{2}}^{*}\right)-\psi\left(d\left(x_{\lambda_{1}}^{*}, x_{\lambda_{2}}^{*}\right)\right)\right] } & +\left[d\left(y_{\lambda_{1}}^{*}, y_{\lambda_{2}}^{*}\right)-\psi\left(d\left(y_{\lambda_{1}}^{*}, y_{\lambda_{2}}^{*}\right)\right)\right] \leq d\left(T_{\lambda_{1}}\left(x_{\lambda_{2}}^{*}, y_{\lambda_{2}}^{*}\right), T_{\lambda_{2}}\left(x_{\lambda_{2}}^{*}, y_{\lambda_{2}}^{*}\right)\right) \\
& +d\left(T_{\lambda_{1}}\left(y_{\lambda_{2}}^{*}, x_{\lambda_{2}}^{*}\right), T_{\lambda_{2}}\left(y_{\lambda_{2}}^{*}, x_{\lambda_{2}}^{*}\right)\right) .
\end{aligned}
$$

Since

$$
T_{\lambda}\left(x_{\mu}, y_{\eta}\right)=e^{-\lambda}-y_{\eta}, x_{\mu}, y_{\eta} \in X, \lambda, \mu, \eta \in[a, b],
$$

then by using Definition 1.1 in (14), we have that

$$
T_{\lambda}\left(\frac{e^{-\lambda}}{3}, \frac{2 e^{-\lambda}}{3}\right)=\frac{e^{-\lambda}}{3}, \text { and } T_{\lambda}\left(\frac{2 e^{-\lambda}}{3}, \frac{e^{-\lambda}}{3}\right)=\frac{2 e^{-\lambda}}{3},
$$

and thus, $\left(x_{\lambda}^{*}, y_{\lambda}^{*}\right)$ is the unique coupled fixed point of $T_{\lambda}$, where $x_{\lambda}^{*}=\frac{e^{-\lambda}}{3}, y_{\lambda}^{*}=\frac{2 e^{-\lambda}}{3}$.

Again, by invoking an elementary result in real analysis- that the sum, or, difference of two continuous functions is continuous, then it is clear from (14) that $T_{\lambda}$ is continuous.

Now, we obtain by using (14) that

$$
T_{\lambda_{1}}\left(x_{\lambda_{2}}^{*}, y_{\lambda_{2}}^{*}\right)=e^{-\lambda_{1}}-y_{\lambda_{2}}^{*}=e^{-\lambda_{1}}-\frac{2 e^{-\lambda_{2}}}{3}, T_{\lambda_{2}}\left(x_{\lambda_{2}}^{*}, y_{\lambda_{2}}^{*}\right)=e^{-\lambda_{2}}-y_{\lambda_{2}}^{*}=\frac{e^{-\lambda_{2}}}{3},
$$

and

$$
T_{\lambda_{1}}\left(y_{\lambda_{2}}^{*}, x_{\lambda_{2}}^{*}\right)=e^{-\lambda_{1}}-x_{\lambda_{2}}^{*}=e^{-\lambda_{1}}-\frac{e^{-\lambda_{2}}}{3}, T_{\lambda_{2}}\left(y_{\lambda_{2}}^{*}, x_{\lambda_{2}}^{*}\right)=e^{-\lambda_{2}}-x_{\lambda_{2}}^{*}=\frac{2 e^{-\lambda_{2}}}{3}
$$

Using (15) gives

$$
d\left(T_{\lambda_{1}}\left(x_{\lambda_{2}}^{*}, y_{\lambda_{2}}^{*}\right), T_{\lambda_{2}}\left(x_{\lambda_{2}}^{*}, y_{\lambda_{2}}^{*}\right)\right)=\left|T_{\lambda_{1}}\left(x_{\lambda_{2}}^{*}, y_{\lambda_{2}}^{*}\right)-T_{\lambda_{2}}\left(x_{\lambda_{2}}^{*}, y_{\lambda_{2}}^{*}\right)\right|=\left|e^{-\lambda_{1}}-e^{-\lambda_{2}}\right| .
$$


Using (16) yields

$$
d\left(T_{\lambda_{1}}\left(y_{\lambda_{2}}^{*}, x_{\lambda_{2}}^{*}\right), T_{\lambda_{2}}\left(y_{\lambda_{2}}^{*}, x_{\lambda_{2}}^{*}\right)\right)=\left|T_{\lambda_{1}}\left(y_{\lambda_{2}}^{*}, x_{\lambda_{2}}^{*}\right)-T_{\lambda_{2}}\left(y_{\lambda_{2}}^{*}, x_{\lambda_{2}}^{*}\right)\right|=\left|e^{-\lambda_{1}}-e^{-\lambda_{2}}\right| .
$$

By using (17) and (18) in the Inequality ( $\star \star$ ) (and with the usual metric on $\mathbb{R}$ ), we have

$$
\begin{aligned}
{\left[\left|x_{\lambda_{1}}^{*}-x_{\lambda_{2}}^{*}\right|-\psi\left(\left|x_{\lambda_{1}}^{*}-x_{\lambda_{2}}^{*}\right|\right)\right]+\left[\left|y_{\lambda_{1}}^{*}-y_{\lambda_{2}}^{*}\right|-\psi\left(\left|y_{\lambda_{1}}^{*}-y_{\lambda_{2}}^{*}\right|\right)\right] } & \leq\left|T_{\lambda_{1}}\left(x_{\lambda_{2}}^{*}, y_{\lambda_{2}}^{*}\right)-T_{\lambda_{2}}\left(x_{\lambda_{2}}^{*}, y_{\lambda_{2}}^{*}\right)\right| \\
& +\left|T_{\lambda_{1}}\left(y_{\lambda_{2}}^{*}, x_{\lambda_{2}}^{*}\right)-T_{\lambda_{2}}\left(y_{\lambda_{2}}^{*}, x_{\lambda_{2}}^{*}\right)\right| \\
& =2\left|e^{-\lambda_{1}}-e^{-\lambda_{2}}\right| .
\end{aligned}
$$

Therefore,

$$
\begin{aligned}
& {\left[\left|x_{\lambda_{1}}^{*}-x_{\lambda_{2}}^{*}\right|-\psi\left(\left|x_{\lambda_{1}}^{*}-x_{\lambda_{2}}^{*}\right|\right)\right]+\left[\left|y_{\lambda_{1}}^{*}-y_{\lambda_{2}}^{*}\right|-\psi\left(\left|y_{\lambda_{1}}^{*}-y_{\lambda_{2}}^{*}\right|\right)\right]=\left[\left|x_{\lambda_{1}}^{*}-x_{\lambda_{2}}^{*}\right|-\frac{3}{4}\left|x_{\lambda_{1}}^{*}-x_{\lambda_{2}}^{*}\right|\right]} \\
& +\left[\left|y_{\lambda_{1}}^{*}-y_{\lambda_{2}}^{*}\right|-\frac{3}{4}\left|y_{\lambda_{1}}^{*}-y_{\lambda_{2}}^{*}\right|\right] \\
& \leq 2\left|e^{-\lambda_{1}}-e^{-\lambda_{2}}\right|
\end{aligned}
$$

that is,

$$
\left|x_{\lambda_{1}}^{*}-x_{\lambda_{2}}^{*}\right|+\left|y_{\lambda_{1}}^{*}-y_{\lambda_{2}}^{*}\right| \leq 8\left|e^{-\lambda_{1}}-e^{-\lambda_{2}}\right| \rightarrow 0 \text { as } \lambda_{2} \rightarrow \lambda_{1},
$$

from which it follows that

$$
\left|x_{\lambda_{1}}^{*}-x_{\lambda_{2}}^{*}\right| \rightarrow 0 \text { as } \lambda_{2} \rightarrow \lambda_{1} \Longrightarrow\left|U\left(\lambda_{1}\right)-U\left(\lambda_{2}\right)\right| \rightarrow 0 \text { as } \lambda_{2} \rightarrow \lambda_{1},
$$

and that

$$
\left|y_{\lambda_{1}}^{*}-y_{\lambda_{2}}^{*}\right| \rightarrow 0 \text { as } \lambda_{2} \rightarrow \lambda_{1} \Longrightarrow\left|V\left(\lambda_{1}\right)-V\left(\lambda_{2}\right)\right| \rightarrow 0 \text { as } \lambda_{2} \rightarrow \lambda_{1}
$$

Hence, the mappings $U, V:[a, b] \rightarrow X$ are continuous.

\section{Conclusion}

So far, we have established some results on the continuous dependence (or, stability) of the coupled fixed points for a distinct set of contractive inequality conditions. To the best of our knowledge, the results contained in the present article are new and original.

\section{REFERENCES}

[1] S. Banach, Sur les operations dans les ensembles abstraits et leur applications aux equations integrales, Fund. Math. 3 (1922) 133-181.

[2] T.G. Bhaskar, V. Lakshmikantham, Fixed point theorems in partially ordered metric spaces and applications, Nonlinear Anal.: Theory Meth. Appl. 65 (2006) 1379-1393. https://doi.org/10.1016/j .na.2005. 10.017.

[3] V. Berinde; On the Stability of Some fixed Point Procedures, Bul. Științ. Univ. Baia Mare, Ser. B. Math. Inform. 8 (2002) 7-14.

[4] V. Berinde, Iterative approximation of fixed points, Springer-verlag, Berlin, Second edition (2007).

[5] L. Ciric, V. Lakshmikantham, Coupled random fixed point theorems for nonlinear contractions in partially ordered metric spaces, Stoch. Anal. Appl. 27 (2009) 1246-1259. https://doi.org/10.1080/ 07362990903259967.

[6] L. Ciric, M.O. Olatinwo, D. Gopal, G. Akinbo, Coupled fixed point theorems for mappings satisfying a contractive condition of rational type on a partially ordered metric space, Adv. Fixed Point Theory, 2 (2012) 1-8

[7] A.M. Harder, T.L. Hicks, Stability results for fixed point iteration procedures, Math. Japon. 33 (1988) 693-706. 
[8] M.O. Olatinwo, Some results on the continuous dependence of the fixed points in normed linear space, Fixed Point Theory 10 (2009) 151-157.

[9] M.O. Olatinwo, On the continuous dependence of the fixed points for $(\varphi, \psi)$-contractive type operators, Krag. J. Math. 34 (2010) 91-102.

[10] M.O. Olatinwo, Some results on the continuous dependence of the fixed points for Kirk-type iterative processes in Banach space, Ann. Univ. Ferrara 56 (2010) 53-63.

[11] M.O. Olatinwo, Coupled fixed point theorems in cone metric spaces, Ann. Univ. Ferrara 57 (2011) 173-180.

[12] M.O. Olatinwo, Stability of coupled fixed point iterations and the continuous dependence of coupled fixed points, Commun. Appl. Nonlinear Anal. 19 (2012) 71-83.

[13] M.O. Olatinwo, The continuous dependence of the fixed points for nonexpansive and quasi-nonexpansive mappings in uniformly convex Banach space, Fixed Point Theory 17 (2016) 427-434.

[14] B. E. Rhoades, Some fixed point iteration procedures, Int. J. Math. Math. Sci. 14 (1991) 1-16.

[15] I.A. Rus, B. Rus, Data dependence of the fixed points set of weakly Picard operators, Stud. Univ. BabesBolyai Math. 43 (1998) 79-83.

[16] I.A. Rus, S. Muresan, Data dependence of the fixed points set of weakly Picard operators, Tiberiu Popoviciu Sem. Cluj- Napoca (2008) 79-83.

[17] I.A. Rus, Generalized contractions and applications, Cluj University Press, Cluj Napoca (2001).

[18] I.A. Rus, A. Petrusal, G. Petrusal, Fixed point theory, 1950-2000, Romanian Contributions, Home of the Book of Science, Cluj Napoca (2002).

[19] I. A. Rus, The theory of metrical fixed point theorem: Theoretical and applicative relevances, Fixed Point Theory 9(2)(2008), 541-559.

[20] F. Sabetghadam, H.P. Masiha, A.H. Sanatpour, Some coupled fixed point theorems in cone metric spaces, Fixed Point Theory Appl. 2009 (2009) Article ID 125426. https://doi.org/10.1155/2009/125426.

[21] T. Zamfirescu, Fixed point theorems in metric spaces, Arch Math. (Basel) 23 (1972), 292-298.

[22] E. Zeidler; Nonlinear functional analysis and its applications-fixed point theorems I, Springer-Verlag, New York Inc. (1986). 